\title{
Psychotherapy Training Experience of General Psychiatrists in the North West Region
}

Diane James, Senior Registrar, Prestwich Hospital, Manchester and Sidney Benjamin, Senior Lecturer, Department of Psychiatry, University of Manchester; Honorary Consultant, Manchester Royal Infirmary

Fifteen years ago the College published guidelines for the training of general psychiatrists in psychotherapy ${ }^{1}$ and these have now been reconsidered in the light of subsequent developments. (Bulletin, October 1986, 10, 286-289). However there is considerable doubt as to the extent to which the previous guidelines have been effective and those responsible for training schemes should consider whether they are providing or can provide the training suggested.

There have been discussion and detailed evaluation of issues such as methods of teaching psychotherapy, e.g., in the Department of Psychiatry in Manchester ${ }^{2}$. of the need for personal psychotherapy ${ }^{3}$ and the attitudes of general psychiatrists to psychotherapy supervision ${ }^{4}$. Attempts to determine what training is received ${ }^{5.6}$ indicate that this probably falls far short of the previous guidelines. It is clear that this is an area where frank responses or any response at all may be difficult to obtain and an attempt to investigate similar issues in two regions in Ireland resulted in a virtually zero response rate. ${ }^{7}$ If guidelines are to be followed it appears essential, as a preliminary step, to find out more about the training actually received.

In April 1985 we sent questionnaires, with stamped, addressed envelopes for their return, to all psychiatric trainees in the North West Region, asking in detail about teaching received and clinical experience in various aspects of psychotherapy. We included consultants appointed in the preceding year who had completed their training in this region. Trainees were assured of anonymity and were offered the choice of including their names on the questionnaire if they wished. One month later a further request was sent to those from whom we had not received an identified, completed questionnaire. Copies of the questionnaire are available from Dr S. Benjamin.

One hundred and fifty-five questionnaires were sent out and 90 replies received; a response rate of $58 \%$. However, nine of these trainees had received most of their training outside the North West Region and we have excluded them from this report. Of the remainder, 34 were Senior House Officers, 32 were registrars and 15 were senior registrars or consultants; 22 had received less than one year of postgraduate psychiatric training, 16 had completed one year, 11 had completed two years, and 32 had completed three years or more.

Seventy replies were from identified trainees and of these two-thirds $(n=47)$ were drawn from teaching hospital trainees. Even if all anonymous responders $(n=20)$ are assumed to be based in non-teaching hospitals, this would still represent a better response rate from those based in teaching hospitals $(69 \%)$ than from those in non-teaching hospitals (49\%), the difference being statistically significant.

We summarise here the training described by the 32 trainees (referred to as 'senior trainees') who had completed at least three years' training in psychiatry, the period for which the College has provided guidelines. After that time trainees are expected to be eligible to enter the Membership Examination and this is clearly a crucial stage at which to review their experience. By this time they have had the opportunity to attend the psychotherapy teaching module in the Membership course provided by the University Department: the course includes a total of 33 lectures and seminars relevant to the theory and practice of psychotherapy. It is improbable that many of those who have received little or no training in psychotherapy by this stage will start to do so subsequently.

\section{Teaching}

Trainees were asked to indicate the number of lectures they had attended on 'learning theory' and the 'theory of psychodynamics' (we use a broad definition of these terms), but as we accepted that not all would consider this an essential form of teaching, we also asked whether 21 specific topics had ever been considered in any type of formal tuition. It is, of course, likely that there will be errors in trainees' recall of teaching, but we think that if teaching of these factual topics cannot be remembered it is unlikely that it made a great impact on their training. Of the 32 'senior' trainees, eight had attended fewer than three lectures or seminars on learning theory and seven had attended fewer than three on psychodynamic theory. None of the specified topics had been covered by all trainees and there were some notable omissions. For example, no teaching was received by four students on desensitization, by eight on relaxation, by 10 on psychodynamic interpretation, by 11 on supportive psychotherapy, by 14 on cognitive therapy and by 18 on marital therapy.

\section{Clinical experience}

Supervised experience of treating patients is the core of psychotherapy training. We therefore asked in detail about patients treated by behaviour therapy, individual psychodynamic psychotherapy and group or family therapy. Of the 32 'senior' trainees, 11 had not treated any patient with individual psychodynamic therapy, but 20 had treated two or more patients. Twelve trainees had no experience of group or family therapy, but 16 had treated two or more groups or families. With regard to behaviour therapy, 14 had never treated any patient, and only 10 had treated two or more patients. Thus approximately one-third had no experience of each of these forms of treatment. There was considerably more experience of individual psychodynamic psychotherapy than of behaviour therapy. 


\section{Supervision}

About two-thirds of these trainees were satisfied with supervision received for the treatment of at least one patient in psychodynamic psychotherapy but only one-third were satisfied with supervision of a patient treated with behaviour therapy. An interesting comment found in three replies was that it was difficult to receive psychotherapy supervision from the consultant for whom one worked and another two trainees commented on the inappropriateness of being supervised by consultants who were obviously not interested in psychotherapy!

We asked about supervision using videotaping of interviews and found only $16 \mathrm{had}$ had any experience of this kind of training.

\section{Personal experience}

Views on the need for personal experience vary greatly, so we asked about a wide range. Of the 23 with personal experience, 13 had attended experiential groups, 13 had attended staff groups, two were in personal analysis and 11 had 'other' experience. Most of the latter was based on attendance at courses run by the regional psychotherapy services at Brindle Lodge. Nine of the trainees had had no personal training experience relevant to psychotherapy.

\section{Satisfaction}

Satisfaction cannot be accurately assessed by this type of survey, but we did ask trainees whether they had been able to use the training they knew to be available and for their general comments. Some thought that they received insufficient guidance and encouragement in the early stages of training and were not aware of the need for psychotherapy experience until it was too late, even though, to others, it appeared readily available. Approximately two-thirds thought they had been able to use the opportunities available, but others commented on the difficulties of finding time for psychotherapy experience in addition to the time needed for attendance at formal courses.

We noted that seven of those who had never treated a patient by psychodynamic psychotherapy claimed to be happy with their training and that the same number of those without any behavioural experience did not see this as a deficit. Thus we have identified a group of trainees who are either unaware of the deficiencies in their training or have positively rejected these areas of experience.

Comments from the total group $(n=81)$ were also of interest. Despite attempts to provide structured introductory teaching in the Membership course, several trainees still stressed the difficulties of embarking on psychodynamic psychotherapy. They asked for a structured training programme that could have introduced them to a supervisor and suitable patient at an appropriate point in their training. Even when trainees took initiative by approaching psychotherapists, they sometimes found that no patient was available for supervised treatment. It is not surprising that in these circumstances one trainee said "It is too easy to defer psychotherapy experience". Group therapy was also seen as an area where training was left too much to the initiative of the individual trainee.

The geographical problems when working in peripheral hospitals were also stressed. It was stated by many trainees in non-teaching hospitals that they felt particularly isolated from psychotherapy training. The consultant psychotherapists, who are dispersed through the region, had offered the clinical tutors arrangements to remedy this problem about one year previously and had received little response. Three health districts contain psychotherapy departments providing a regional service and four others have specialist psychotherapy services. It appears that it is more difficult to obtain experience in some parts of the Region than in others, but the reasons for this remain obscure.

\section{Experience and training grade}

The results from all respondents, whatever their length of training, showed that a greater proportion of those in their third year $(n=10)$ had experience in some areas than did those with at least three completed years. This prompted us to examine the latter group according to their training grade. The 15 senior registrars and newly appointed consultants differed notably from the 17 senior house officers and registrars. Both groups have little experience of behavioural psychotherapy. However, all except one of the senior registrar and consultant group had treated at least three patients with psychodynamic techniques, whereas only six of the 17 SHOs and registrars had done so. Most of the senior registrar group had had teaching in most of the specific topics, but few of the SHOs and registrars had covered even the most important topics. For instance only four of the $17 \mathrm{had}$ had teaching on marital therapy and only eight on transference. Approximately 10 of our 81 respondents have had three or more years of experience of psychiatry but little training in psychotherapy and have remained in junior grades.

\section{Discussion}

In a survey of the psychotherapy experience of 81 psychiatry trainees in the North West Region we have focused particularly on a group of 32 who have completed at least three years' training. A substantial minority appear to have received considerably less supervised practical experience or theoretical instruction than that proposed by the College 15 years ago. One-third have attended fewer than three lectures on each of the entire fields of learning theory and psychodynamic theory. A similar or greater proportion have received no teaching of any kind on topics such as relaxation, marital therapy and supportive psychotherapy. About half the group have no experience of using behavioural psychotherapy and about one-third none of individual and group or family techniques. Those who remain in the registrar and SHO grade after at least three years' training usually show deficiencies in all aspects of psychotherapy training and many of these are apparently unaware of, or are satisfied with, this deficit. Those who have attained senior registrar grade have generally received adequate teaching and good experience in individual 
psychotherapy (judged by quantity rather than quality), but even these have relatively little experience of behavioural techniques.

The comments made by the whole group of trainees appear helpful in explaining this. Seven trainees commented at length on the lack of experience in behavioural methods. Comments included "There is little training in these therapies and little encouragement to undertake them"; "No one suggested I should take on a patient for behaviour therapy"; "Opportunities to do behaviour therapy are nil due to the closed shop operated by clinical psychologists". One commented that it was not until she had completed three years' training that it occurred to her that she needed training in behavioural psychotherapy, and another that "behaviour therapy should be demystified". In contrast, there were many favourable comments about the quality of psychodynamic experience.

We had replies from just over half the trainees in the North West Region. We cannot compare our respondents with non-respondents, as we had many anonymous replies. However, it seems unlikely that trainees who failed to respond to our questionnaire have more interest or experience in psychotherapy than those who did respond, and it is possible that a better response rate would have revealed greater deficiencies. The response rate was almost as good as those achieved in the best of the other surveys cited above and their results reveal that our findings are likely to reflect wide-spread rather than local problems.

This survey was designed to study aspects of training actually received but some tentative conclusions can also be drawn about possible ways of improving training, based particularly on trainees' comments. Guidance about the need for psychotherapy training, its range and the facilities available should be given to all trainees at the time that they start general psychiatry training. It should be provided by the regional psychotherapy service, as well as by clinical tutors and other consultants, to ensure that it is uniformly available. Trainees should be provided with suitable patients and supervision within a structured programme once they have taken the initiative to obtain clinical experience. This may help to break the cycle of apathy or antagonism into which some trainees find themselves drawn (and perhaps to which some are predisposed). It is not evident whether an increase in training resources would reach a greater proportion of trainees or whether it would only improve the experience of those who are already receiving good training; there is no doubt that training opportunities that are available are not fully used. However it is difficult to obtain supervised experience of behaviour therapy: there is no consultant psychiatrist with special responsibility for behavioural psychotherapy in the Region and clinical psychologists, perhaps understandably, appear to give priority to training students in their own profession.

\section{ACKNOWLEDGEMENTS}

We would like to thank Professor David Goldberg, Dr Frank Margison and Dr John Kincey for their helpful comments on the manuscript.

\section{ReFERENCES}

'The Royal Coliege of Psychiatrists (1971) Guidelines for the training of general psychiatrists in psychotherapy. British Journal of Psychiatry, 119, 555-557.

${ }^{2}$ Magutre, G. P., Goldberg, D. P., Hobson, R. F. et al. (1984) Evaluating the teaching of a method of psychotherapy. British Journal of Psychiatry, 144, 575-580.

${ }^{3}$ Cox, J., Marks, I., Marteau, L. \& Steiner, J. (1982) Personal psychotherapy in the training of a psychiatrist? Bulletin of the Royal College of Psychiatrists, March, 6, 38-42.

${ }^{4}$ Lieberman, S. \& CoBb, J. (1983) Psychotherapy supervision: the attitudes of general psychiatrists. Bulletin of the Royal College of Psychiatrists, June, 102-103.

so'Shea, B., Cahill, M. \& McGennis, A. (1983) Traince psychiatrists: attitudes to psychotherapy and its training. Irish Journal of Psychotherapy, 2, 10-13.

${ }^{6}$ CahllL, M., O'SheA, B. \& MCGennis, A. (1983) Trainee psychiatrists: attitudes to psychotherapy and its training in Northern Ireland. Irish Journal of Psychotherapy, 2, 75-77.

'O'Shea, B., CAhILL, M. \& MCGennis, A. (1986) If at first you don't succeed... Bulletin of the Royal College of Psychiatrists, January, 10, 12-13.

\section{The Role of the Hypothalamo Pituitary Adrenal Axis in Psychiatry}

A one-day meeting 'The Role of the HPA Axis in Psychiatry', preceding the British Association for Psychopharmacology meeting, will be held at the Tenovus Institute, Cardiff on 19 February 1987. Speakers include Professor F. Holsboer, Dr N. Ferrier, Dr M. Scanlon and Dr
D. Riad-Fahmy. Completed application forms must be received by the workshop secretary no later than Monday, 19 January 1987. They may be obtained from: Dr Brian Harris, Department of Psychiatry, Sully Hospital, Penarth, Cardiff (telephone 0222 530266, extension 5738). 